\title{
A review of current theories and treatments for phantom limb pain
}

\author{
Kassondra L. Collins, ${ }^{1}$ Hannah G. Russell, ${ }^{2}$ Patrick J. Schumacher, ${ }^{2}$ Katherine E. Robinson-Freeman, ${ }^{2}$ Ellen C. O'Conor, ${ }^{2}$ \\ Kyla D. Gibney, ${ }^{2}$ Olivia Yambem, ${ }^{2}$ Robert W. Dykes, ${ }^{3}$ Robert S. Waters, ${ }^{1}$ and Jack W. Tsao ${ }^{2,4,5}$
}

'Department of Anatomy and Neurobiology and 'Department of Neurology, University of Tennessee Health Science Center, Memphis, Tennessee, USA. ${ }^{3}$ School of Physical and Occupational Therapy, McGill University, Montreal, Quebec, Canada. ${ }^{4}$ Department of Neurology, Memphis Veterans Affairs Medical Center, Memphis, Tennessee, USA. ${ }^{5}$ Children's Foundation Research Institute, Le Bonheur Children's Hospital, Memphis, Tennessee, USA.

\begin{abstract}
Following amputation, most amputees still report feeling the missing limb and often describe these feelings as excruciatingly painful. Phantom limb sensations (PLS) are useful while controlling a prosthesis; however, phantom limb pain (PLP) is a debilitating condition that drastically hinders quality of life. Although such experiences have been reported since the early 16th century, the etiology remains unknown. Debate continues regarding the roles of the central and peripheral nervous systems. Currently, the most posited mechanistic theories rely on neuronal network reorganization; however, greater consideration should be given to the role of the dorsal root ganglion within the peripheral nervous system. This Review provides an overview of the proposed mechanistic theories as well as an overview of various treatments for PLP.
\end{abstract}

\section{Introduction}

Amputations cause changes in both the PNS and CNS, including the emergence of phantom limb sensations (PLS), characterized by the feeling that the amputated limb is still present. Most amputees experience PLS and can even control phantom movements, such as wiggling toes or opening and closing the hand, immediately after surgery $(1,2)$. The majority of amputees also experience intense episodes of pain throughout the missing limb that are termed phantom limb pain (PLP), characterized by throbbing, stabbing, electric shock sensations, and even cramped and painfully immobile limb sensations (3).

A French surgeon, Ambroise Paré, was likely the first to document an instance of PLP, in the 16th century (4), but the term did not arise until the American Civil War, when it was described by military battlefield surgeon (and later, neurologist) Silas Weir Mitchell (5). Clinicians did not appreciate the high incidence or pathophysiological basis of PLP until recently. They often attributed PLP to psychological problems, especially during the Civil War era $(6,7)$. During World War II, nearly 15,000 US service members lost a limb during combat (8). Many amputees did not publicly share their PLP experiences for fear of being stigmatized as mentally ill (7).

Approximately 1.9 million amputees live in the US, with worldwide projections expected to double by the year 2050 (9). Amputations are commonly a consequence of diabetes mellitus, trauma, and cancer (9). Combat-related limb loss is also a frequent cause of amputation: as of January 1, 2018, 1,718 US military service members had lost at least one major limb in Iraq or Afghan-

Conflict of interest: The authors have declared that no conflict of interest exists. Reference information: J Clin Invest. 2018;128(6):2168-2176.

https://doi.org/10.1172/JCI94003. istan (10). The majority of amputees, but not all, experience PLP to some extent, with varying degrees of severity, frequency, and episode duration. PLP eventually dissipates or disappears in some, while others report no change in frequency or severity (11). Estimates of PLP incidence differ considerably, depending in part on the sampled population as well as the methods of reporting and data collection. In one large, frequently cited survey of amputees, 78\% reported experiencing PLP (12).

Considered a neuropathic pain or "complex pain state of the somatosensory nervous system" (13), PLP is thought to be driven by CNS abnormalities. However, research investigating the contribution of the PNS and its function also needs to be considered (14). While the mechanisms underlying PLP remain unclear, it is known that sensitized and reorganized nerve endings and cell bodies within the peripheral limb affect the CNS, causing changes in somatosensory processing pathways (15). PLP presents a considerable impairment to amputees' quality of life, and a better understanding of its pathophysiology and etiology could lead to new modalities to alleviate the suffering it causes. This Review aims to provide up-to-date knowledge regarding the current state of PLP theories, research, and therapies.

\section{Mechanistic theories of PLP: CNS versus PNS}

Researchers and clinicians continually debate the mechanisms of PLP and the contributions of the CNS and/or the PNS. Currently, the most commonly posited CNS theory is the cortical remapping theory (CRT), in which the brain is believed to respond to limb loss by reorganizing somatosensory maps (16). Early theories focused solely on the contribution of neuromas (abnormal growth or thickening of nerve tissue), although there was evidence of pain immediately after surgery (17). More recent research on the peripheral causes of PLP focuses on the inability of the severed nerves to 
A

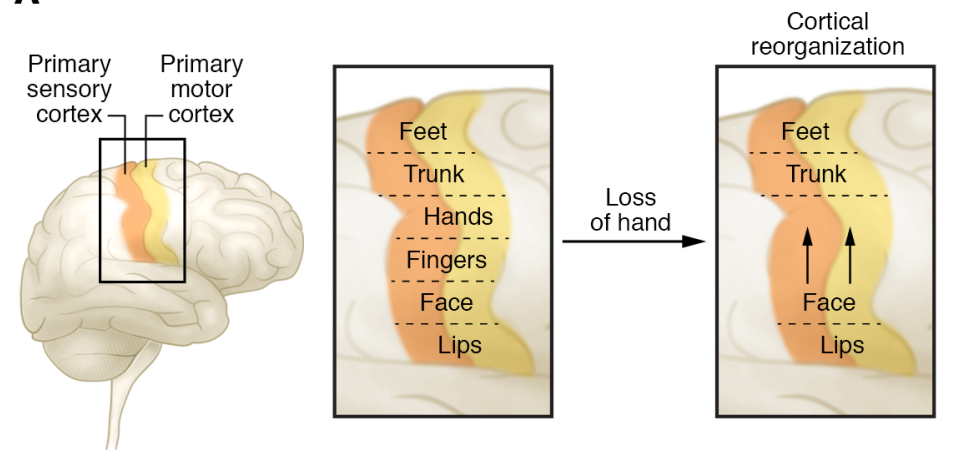

B

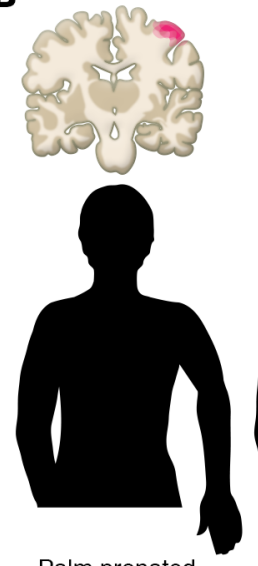

Palm pronated

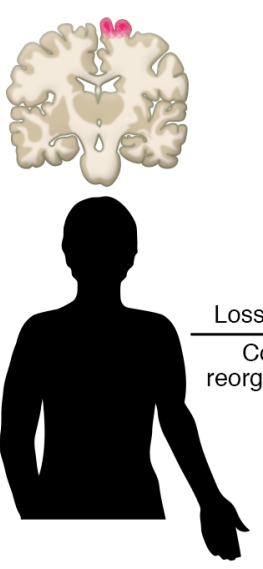

Palm supinated
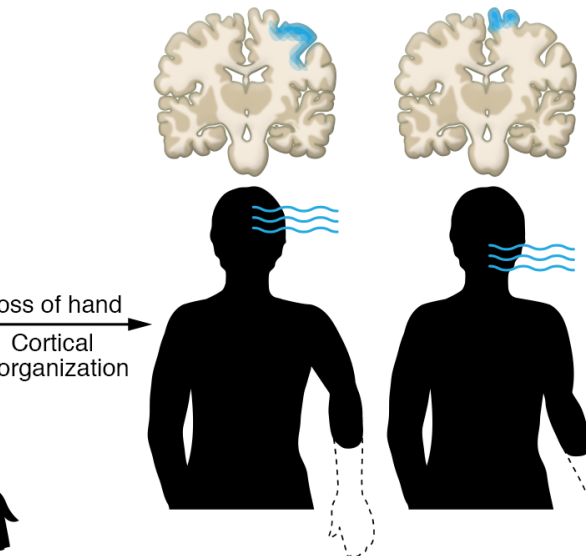

Stimulation to reorganized section of cortex can lead to proprioceptive memory sensation of missing limb
Figure 1. Cortical contributions to PLS and PLP. (A) Body part sensory and motor representation are laid out in a pattern that forms the cortical homunculus and receives sensory information (e.g., tactile, olfactory, or pain) from different areas of the body (24). Following amputation, a cortical region that received sensory or motor projections from the amputated limb may begin to receive sensory or motor input, respectively, from neighboring cortical regions, which expand to take over the region that previously controlled the amputated limb $(27,28)$. (B) Proprioceptive memory, which stores information about the position of the limb in space relative to the body, may influence cortical reorganization in the CNS. These memories may store information about the final position of the missing limb or, in combination with cortical reorganization, may affect PLS or PLP. This image illustrates rapid changes in cortical activation patterns that can occur simply with repositioning of the phantom limb, manifested as changes in the location of hand sensations mapped onto the face. repair previous connections (with or without neuroma formation), the role of the dorsal root ganglion (DRG), and preamputation pain $(18,19)$. While an amputation directly affects the PNS, the CNS is also affected due to changes in sensory and movement signaling. Debate still remains over the cause and maintaining factors of both phantom limbs and the associated pain.

\section{Mechanistic theories of PLP involving the CNS}

Neuromatrix representation. Many of the theories explaining the causation of PLP rely on the concept of a representation of the self within the brain that is modified by life experiences, termed the neuromatrix (19). After limb amputation, an individual's cortical and peripheral body representations remain intact, but no longer correspond, and this mismatch is enhanced by a lack of visual feedback from the missing limb, thus generating excessive pain, in spite of the lack of a sensory stimulus (20). A study investigating the relationship between body representation within a dream and the experience of PLP found a positive correlation between increased PLP after lower limb amputation and the ability to recall intact body representations (21). These findings suggest that aversive somatosensory experiences mediate the skewed interactions between mental and physical body representations, which then facilitate PLP (21).

Research investigating the malleability of the neuromatrix has attempted to determine the ability of the brain to adjust to sensory stimuli. An example is the rubber hand phenomenon, which occurs when an able-bodied person perceives a rubber hand as his or her own. To achieve this effect, the person's own hand is hidden from view, typically under a table, while a rubber hand is put in front of his or her body (22). Both the rubber and the actual hands are synchronously stroked with a brush, causing the person to perceive the rubber hand as his or her own (22). To test rubber hand incorporation into the neuromatrix, the researchers strike the rubber hand with a hammer (23). Participants flinch in fear of pain, even though the actual body part is unharmed (23), demonstrating that the neuromatrix is rapidly malleable/adaptable and is greatly affected by visual representations and somatosensory stimuli.

Although the rubber hand demonstration does not show that conflicts within the neuromatrix cause pain, discomfort similar to that experienced by amputees can be induced in able-bodied volunteers by causing conflict between motor and sensory processes (24). In another study, volunteers moved their upper and lower extremities in a congruent or incongruent fashion while viewing such movements in a mirror or with their view blocked by a whiteboard. The majority of reported symptoms occurred while participants completed incongruent movements while viewing the reflection of the limb in the mirror, causing the most conflict between motor and sensory processes. The symptoms reported included numbness, pins and needles, aching, and uncomfortable pain (24), demonstrating that conflicts among visualization, somatosensory input, and cortical representation may play a role in PLP.

\section{CRT}

The CRT posits that cortical reorganization accounts for the neurophysiological origin of PLP (ref. 17 and Figure 1A). According to the CRT, neurons that received input from an arm 


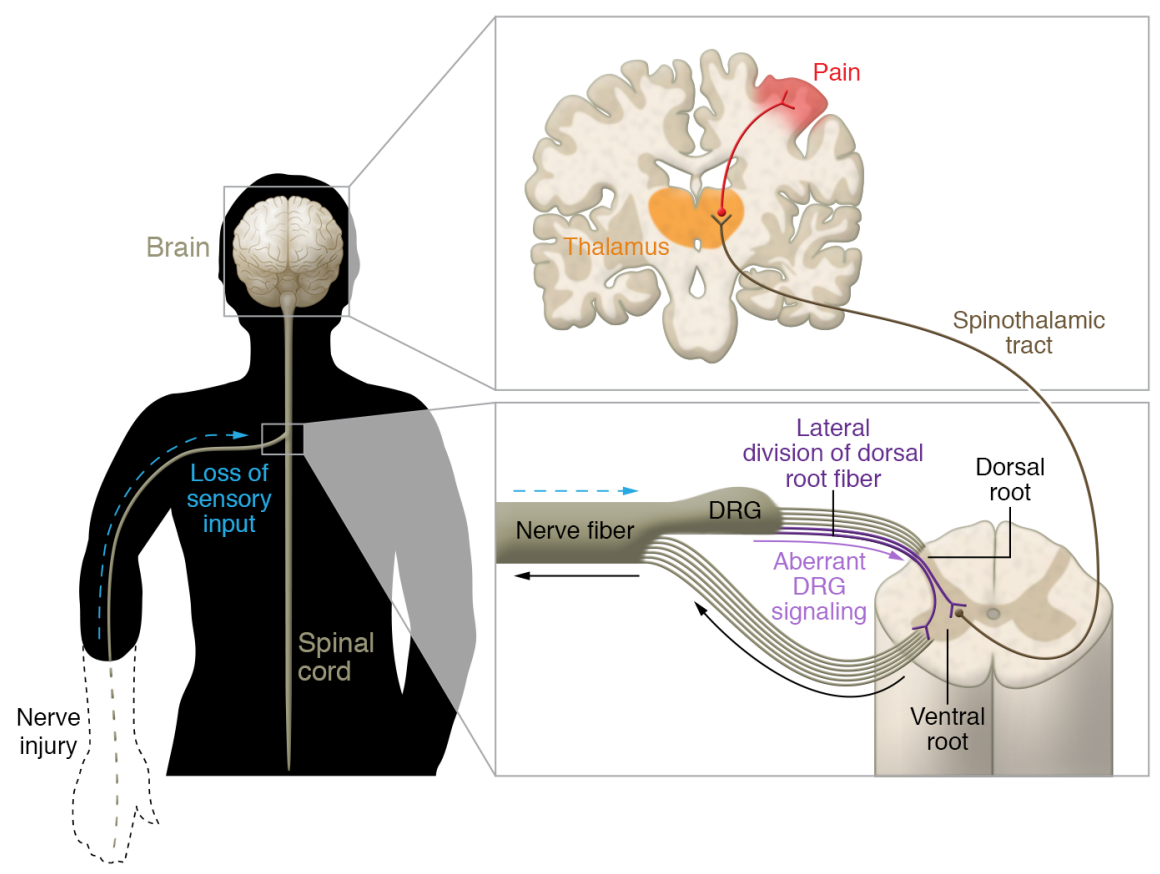

Figure 2. Proposed peripheral contributions to PLS and PLP. The dorsal root fibers of the DRG split into lateral and medial divisions (38). The lateral division sections contain most of the unmyelinated and small myelinated axons and specifically carry pain and temperature information. The medial division sections of the dorsal root fibers (not shown) contain mostly myelinated axons that convey sensory information from the skin, muscles, and joints, such as touch, pressure, proprioception, and vibration (38). When an injury occurs to the nerves, neurons in the DRG increase their nociceptive signaling through increases in neuronal excitability and the creation of ectopic discharges (25). The resulting aberrant signaling through the spinothalamic tract may produce PLP.

before its amputation subsequently respond to new inputs from the face that invade the nearby arm-associated somatosensory region; consequently, with facial stimulation, an amputee may experience PLS, including pain $(11,25)$. Expansion and invasion within the somatosensory cortex have been attributed to a lack of sensory information reaching the cortical area that once controlled the missing limb (26). The mammalian brain is remarkably plastic, and investigations of both simian and human brains have shown somatosensory cortical rearrangement in response to amputation.

Early research on animals using microelectrode-mapping techniques provided evidence for the reorganization of cortical maps in the somatosensory cortex following amputation (27). In their seminal study, Merzenich and colleagues found that after long-term deafferentation from removal of a digit, the neurons in the cortical map of an amputated middle finger started to respond to stimuli applied to the adjacent digits. Similarly, facial sensations in a corresponding area of the phantom hand were detectable 24 hours after amputation in a person, which is suggestive of cortical reorganization (28). In a human functional MRI study, investigators reported that the lip area of the somatosensory cortex contralateral to the side of amputation was located more medial and superior than the lip area contralateral to the intact limb (29). Researchers also observed reduced cortical reorganization after the administration of brachial plexus anesthesia, with the lip area shifting away laterally from the amputation zone (29). Furthermore, under spinal anesthesia, PLS and pain arise in patients who have never experienced PLS or PLP previously (30). Recently, we reported that a man who suffered a brachial plexus avulsion (BPA) (an injury to the nerves of the cervical spinal cord) noted rapid onset of PLS and developed hand-to-face remapping, which reversed following nerve grafting (31). These observations suggest that cortical remapping might be explained by an unmasking of normally dormant synaptic connections or a rapid shift of cortical network connections in addition to the formation of new connections that occurs later with axonal sprouting $(31,32)$. This unmasking could result from a decrease in the number of neurons releasing GABA, the main inhibitory neurotransmitter in the brain, after deafferentation (33). A study of cortical maps found that GABA-mediated inhibition in the motor cortex led to sporadic, involuntary limb movements, suggesting that maintenance of normal GABA levels can suppress cortical reorganization that might lead to PLP (33). Furthermore, GABA levels are also known to fluctuate in the PNS.

Other research, however, argues that the integrity of the excised limbs' cortical map during PLS is maintained due to PLP experiences. A recent series of experiments found no robust relationship between cortical rearrangement and PLP, arguing that many different factors may play roles in maintaining structural, and even functional, capabilities necessary to control phantom limbs (34). Makin's group proposes that both bottom-up (peripheral to central) and top-down (central to peripheral) pain pathways maintain the cortical representation of the limb and facilitate PLP (34). In this research, phantom movements and motor imagery were used to elicit responses instead of sensory stimulation. Such factors may explain the different findings.

The role of the somatosensory cortex in PLP is greatly debated. Penfield extensively studied the brain with electrical stimulation, finding no areas that produced pain, not even the somatosensory cortex (35). However, transcranial magnetic stimulation (TMS) to the sensory cortex has been shown to reduce PLP, demonstrating that the area does play some role in such pain (36). A recent case study reported that upper-extremity PLP relief was achieved in a person after 28 sessions of repetitive TMS (36). More research is needed to determine the mechanisms and causation of PLP and whether changes in the cortex after amputation play a role in pain and/or sensation. 
A

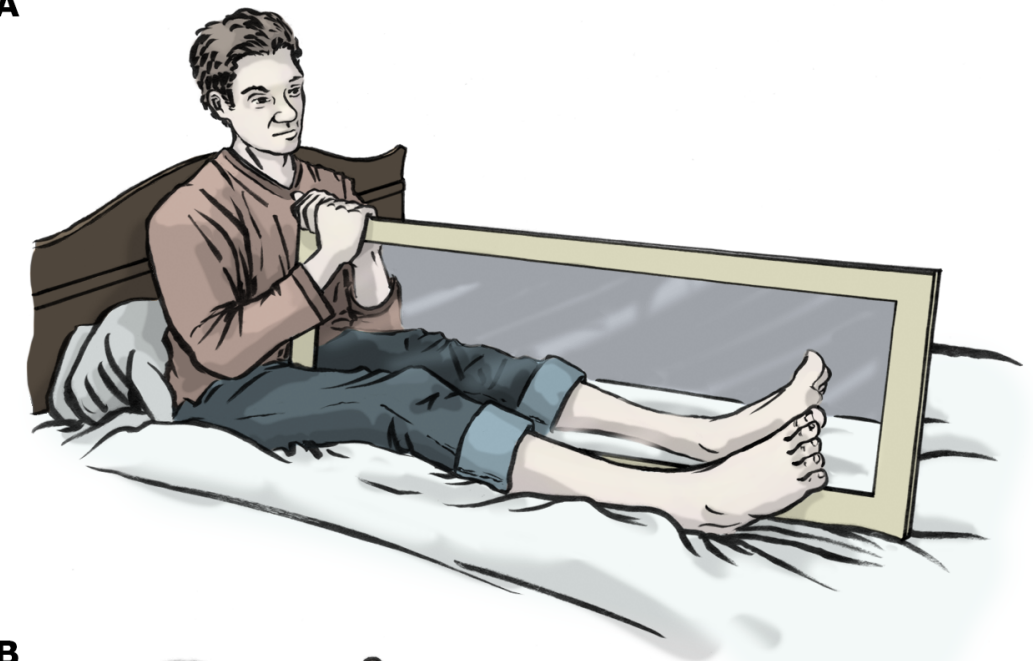

B

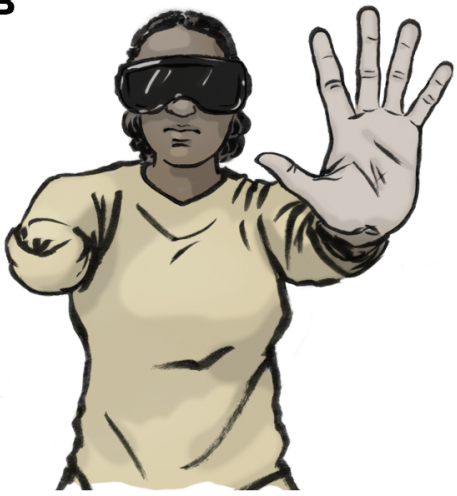

Figure 3. PLP-targeting interventions. (A) MT is a potential treatment option for PLP. In this approach, devised by Ramachandran, an amputee attempts to alleviate PLP by moving his/her intact right limb in front of a mirror to create a visual representation of the missing limb while simultaneously moving the phantom limb (94). Although MT has been shown to be effective at reducing PLP in many, but not all, amputees, the mechanisms of pain reduction are not well understood. MT uses visual feedback of movements by the intact limb to reduce pain, which is crucial to efficacy, as pain reduction was not seen when the mirror was covered with a sheet (75). (B) Similarly to MT, VR therapy relies on visual feedback by simulating both intact and missing limbs. Participants wear VR goggles to visualize a representation of the missing limb.

\section{Subcortical theories: thalamic contributions}

Somatosensory and motor cortices may not be the only areas affected by amputation. Subcortical structures, including the thalamus, may also be reorganized $(15,19,37)$. Changes at the subcortical level may originate in the cortex and cause reorganization through strong efferent connections to the thalamus and lower structures (37). It is also possible that reorganizational processes begin at the thalamic level and changes are relayed up to the cortex (19). In an effort to map the thalamus in amputees, researchers using microstimulation and microelectrode recordings found that the representation of the residual limb in the thalamus was enlarged compared with that of corresponding areas of individuals with intact limbs and that thalamic stimulation could evoke PLS and even PLP in amputees (38).

The thalamus has also been investigated as the sole pain-generating structure. Studies have shown that, following spinal cord injury, hyperexcitability of thalamic neurons is independent of synaptic drive from spinal neurons, suggesting that the thalamus can be transformed into an autonomous pain-signal generator (39). Patients with spinal cord injury often experience PLP and PLS (39). In a rodent model, forelimb amputation resulted in reorganization in both deafferented primary somatosensory cortex and the ventral posterior nucleus of the thalamus, the latter of which relayed the new input to the deafferented cortex (40). This finding lends further credence to a thalamic contribution to cortical reorganization.

\section{Proprioceptive memory}

Another possible mechanism underlying PLP is proprioceptive memory. Proprioception is the brain's awareness of the position of the body's limbs in 3D space. Amputees continue to have proprioception of missing limbs, including both voluntary and involuntary movements. A voluntary movement sensation includes an amputee's attempt to move the phantom limb, while an involuntary movement sensation is the feeling of the limb being frozen or sporadically moving on its own (32). One theory posits that the proprioception needed to perform specific tasks may be incorporated into a "proprioceptive memory" that aids us in accomplishing the tasks more quickly and efficiently in the future (41). When an amputation occurs, memory engrams of the limb are retained even though visual feedback confirms limb absence (Figure 1B). Supporting this theory is a study of limb repositioning after regional anesthesia, with patients reporting that their limbs remained in the last position they remembered before anesthesia (42). It is also possible that proprioceptive memories provide a protective feature, serving as a reminder of painful situations and how to remedy them, such as moving a joint out of hyperextension without having to confirm with visual feedback (41). Thus, certain positional movements with the phantom limb may trigger these painful proprioceptive memories. Amputees have reported feeling their phantom limbs stuck in the last positions they remembered prior to amputation, supporting a stored proprioceptive memory as the final feedback from the limb (43). 


\section{Dissociation of vision and proprioception}

Limb movement typically relies on both visual and proprioceptive systems working together. For instance, vision primarily guides hand movements toward a target. While the hand is moving, the brain receives proprioceptive feedback regarding the location of the limb relative to the body. The brain coordinates each piece of information to complete the directed movement. With an amputation, visual feedback of the now-removed limb is no longer available. However, proprioception regarding the location of the once-intact limb still remains, either through proprioceptive memories or activation from the residual limb nerve endings. Perhaps the inability to visualize the amputated limb is insufficient to override the proprioceptive information from the residual limb. An alternative possibility is that the brain's interpretation of conflicting signals from the two systems resurrects a phantom limb. The fact that visualization therapies have been relatively successful at reducing PLP implies that the accuracy provided by both visualization and proprioception may be critical in reducing PLP (44).

\section{Theories involving the PNS}

Unlike the well-protected CNS, the PNS is highly susceptible to injury. Early research focused on the PNS as the sole cause and maintenance factor of PLP. However, peripheral factors alone cannot mediate the emergence of PLP (19); rather, the PNS may work in conjunction with the CNS to cause and maintain the persistence of PLP. There is much debate over whether "top-down" or "bottom-up" maintenance is the cause of PLP. Bottom-up pain mechanisms imply that peripheral nerve injury causes excessive aberrant inputs that, in turn, influence changes (or lack thereof) in the cortex $(12,34)$. Top-down pain modulation refers to painful sensations that are maintained by the CNS and are greatly affected by emotional state, memories, and attention (45). Some regions of the brain experience reorganizational changes after an amputation. Therefore, further research investigating the changes that occur within the residual limb after an amputation, the effects of peripheral changes on the CNS, and how each effect is maintained are crucial to expanding the knowledge regarding PLP.

\section{Neuromas and the DRG}

The cell bodies for the somatic-component PNS axons are located in the DRG. Neurons in the DRG are PNS afferents, relaying sensory information, such as fine touch, proprioception, and vibration, to the CNS (ref. 46 and Figure 2). The proximal ends of DRG axons terminate in the spinal cord, the target of the somatic component being the superficial layers of the dorsal horn (DH) and the dorsal column nuclei of the brain stem. Following limb amputation, DRG axons are disconnected from their distal targets and inflammation and sprouting occur in the resulting residual limb, where a neuroma can form. Far from becoming silent and idle, the injured axons within the residual limb and remaining segment of the peripheral nerves generate spontaneous activity from ectopic, hyperexcitable loci that are propagated along the remaining pathway to the spinal cord. The electrical activity has been described as ectopic, since it is not coming from the normal end points of the axons. Thresholds are abnormal and action potentials seem to be generated spontaneously or in response to stimuli that normally would not provoke an action potential, such as mechanical stimuli (e.g., Tinel's sign) or circulating substances, such as adrenaline (33). In the past, activity in a neuroma has been considered a possible source for PLP. Anesthetizing the residual limb or neuroma by injection was reported to attenuate or abolish PLP in some, but not all, instances (2, 18, 38, 47-50), subsequently leading to diminished enthusiasm for a peripheral origin hypothesis of pain $(15,29,51)$. Currently, supraspinal central mechanisms receive more attention.

Although the trauma at the site of the injury may elicit local inflammation, the responses of injured axons depend upon the cell body receiving messages from the periphery to alter somatic metabolic machinery and start the repair process. The messages that signal the nature of an injury to the cell body could be a loss of tonic electrical or chemical messages that the amputation removed. These might include lost molecular signals, sometimes known as trophic factors, from end organs or supporting elements that are no longer being sent to the nucleus by axonal transport. Alternatively, the signals could represent the loss of electrical activity that arises from the innervated tissues. Additionally, nerve transection may trigger the central propagation of molecular signals arising from the local inflammatory processes at the site of transection or from action potentials arising from the same site, perhaps secondarily to molecular changes in the environment of the severed axons (52).

The nature and time course of morphological changes in the DRG cell body following axotomy have been documented by a number of investigators (reviewed in ref. 52). Histological and biochemical evidence show that the cellular metabolic machinery is modified dramatically, which is described as a "phenotypic change" in the neuron (53). Although some changes are related to the growth response at the end of the residual limb, other changes occur at the axonal extensions into the spinal cord dorsal root. Modifications of the central terminals of transected axons could induce further "phenotypic changes" in the postsynaptic neurons, and the surrounding supporting elements and hundreds or even thousands of gene and protein changes occur in the transected neurons (52). Changes in the DH begin within minutes after the pattern of sensory input changes; central sensitization also begins within minutes (54), and the amplitude of the spinal reflex changes (55). It is likely that similar alterations in neuronal responsiveness occur centrally within minutes of nerve transection.

DRG soma express receptors for acetylcholine (56), glutamate $(57,58)$, and GABA $(59)$ in quantities sufficient to have strong neuromodulatory effects on sensory signaling. The receptors' presence raises two issues. What could be the source of the substances that activate these receptors in the DRG, and what is the impact of their activation? Activation of the somatic GABA receptors may gate nociceptive transmission, but even more complex neuromodulatory effects are possible (59).

Amputation or nerve transection changes the distribution of the receptors on DRG cell bodies. Those alterations may play an important role in various forms of chronic pain, including PLP, and lend credence to the hypothesis (53) that DRG neuronal cell bodies are the source of electrical activity that drives neuropathic pain and PLP (60). Potential determinants of DRG neuronal hyperactivity include, but are not limited to, upregulation of voltage-gated sodium channels (61-63), downregulation of potassium channels $(64,65)$, increased expression of neurotropic factors (66), 
and sprouting of sympathetic noradrenergic axons into the DRG (67). Over the course of the neuronal response to nerve transection, thousands of genes are either upregulated or downregulated, suggesting a potentially large list of gene products that might alter neuronal behavior after nerve transection (68).

If the PNS is the sole contributor to PLP, then it should be possible to induce anesthesia into the limb and eliminate the experience of PLP. Local anesthesia directly injected into the residual limb of amputees experiencing PLP does not lead to reduced PLP in all instances (29). However, changes within the PNS may affect the amount of cortical reorganization experienced. Even if a neuroma does not form, the nerve fibers within the residual limb can undergo spontaneous sprouting and seek new connections. Such random connections may lead to abnormal CNS feedback, resulting in modulation of cortical reorganization and the experience of PLP (19).

There is solid evidence to support the notion that the formerly unappreciated PNS, and DRGs in particular, may be important drivers of PLP and PLS. While we still do not understand the mechanisms underlying PLP, the PNS must now be considered a viable component of any theory of PLP. Currently, there are hundreds of theories in the literature, and few or none are capable of being tested rigorously. The new approaches demonstrated by Devor and colleagues (60) may help the development of testable theories able to eliminate alternative explanations.

\section{Preamputation pain}

Studies have shown that persons who experienced pain prior to amputation have higher rates of $\operatorname{PLP}(18,69,70)$. These studies, however, find no evidence that preamputation pain plays a role in persistent PLP, only PLP experienced immediately after surgery. For instance, one prospective study of 58 patients undergoing an amputation showed that $72 \%$ of those with preamputation pain experienced PLP eight days after amputation, which decreased to $65 \%$ at six months and $59 \%$ after two years (18). However, the location and characterization of the pain was only similar to that experienced before amputation in $10 \%$ of patients (18).

\section{Role of prostheses}

The correlation among cortical reorganization, the experience of PLP, and daily prosthesis usage has also been studied, with daily prosthesis usage found to be hindered by both the amount of cortical reorganization and the cumulative amount of PLP experienced (71). A study of a small number of amputees found those who experienced PLP demonstrated more excitable motor cortex areas and greater reorganization within the areas of the somatosensory cortex that represent the tongue and amputated limb (71). These findings suggest that somatosensory reorganization is correlated with PLP and that such reorganization may cause a secondary reorganization in the motor cortex (71). Motor reorganization and PLP severity were found to be negatively correlated with prosthesis usage, implying that the more an amputee uses the prosthesis, the less reorganization and PLP occur (71). Questions do arise, however, such as the following. Does wearing a prosthesis reduce cortical reorganization, which in turn reduces PLP? Or are those amputees who experience less cortical reorganization the ones who are more likely to use a prosthesis? Further, does the act of using the residual limb to control the prosthesis affect PLP?
A recent study examined PLP in nine BPA patients and one hand amputee using prostheses controlled by a brain-machine interface (BMI) (72). This study found that altering the plasticity of the cortical representation of the phantom hand drastically altered the associated PLP. However, in direct opposition to the ideas postulated by the CRT, increasing the phantom representation increased PLP, whereas increasing the representation of the intact hand reduced PLP, suggesting that BMI training aimed at dissociating the phantom hand from the prosthesis could be a clinically advantageous treatment for PLP (72). Many of the questions mentioned above also apply to the relationship between treating PLP using either mirror therapy (MT) or virtual reality (VR) and prosthesis usage. Preißler and colleagues recently investigated plasticity in the ventral visual streams in relationship to prosthesis usage, postulating that the observed plasticity is related to functional prosthesis use that provides increased visual feedback to the user, which is necessary for controlling the device (73). The study initially did not find a simple correlation between PLP experiences and prosthesis usage. However, a subanalysis revealed that the group experiencing high PLP rates (severity indicated on a visual analog scale) spent less time using prostheses. Amputees experiencing high amounts of PLP and with high prosthesis usage had smaller posterior parietal cortices than patients who did not use prostheses (73). Variability in the posterior parietal cortex volumes indicates that prosthesis use may drive adaptations that lead to changes within the visual stream (73). Without a somatosensory component associated with prosthesis usage, visualization is crucial and may enable changes in PLP experiences similar to MT.

A 2007 study examining the roles of vision and kinesthetic information in proprioception found that vision is more influential in regard to spatial location of a limb (74). During this study, participants experienced tendon vibration to cause the feeling of flexion movements of a limb that was immobilized. When the participants' eyes were closed, they reported feelings of slow movement due to the vibrations. In contrast, if the participants viewed their static vibrating limbs, the perception of movement was drastically hindered, with functional imaging revealing activity in the posterior parietal cortex correlated to the attenuation of movement (74). These findings imply that the posterior parietal cortex plays a role in overcoming kinesthetic proprioceptive information when visual information is provided. Thus, from the experimental evidence, it seems reasonable to conclude that MT, VR, and prosthesis usage all may play a role in diminishing PLP by enabling the amputee to visualize a limb moving in a natural manner. However, each of these methods involves the activation of the residual limb muscles, the role of which in the reduction of PLP remains to be determined.

\section{Treatments for PLP}

The most commonly administered pharmacological treatments for PLP are gabapentin and pregabalin, antiseizure medications that reduce the frequency and intensity of neuropathic pain (75). Opioids and opiates have long been used to treat neuropathic pain as well, and some research suggests that they are effective at ameliorating the symptoms of PLP $(76,77)$. Opioids may relieve PLP by reducing cortical reorganization in the somatosensory cortex (78). Despite their effectiveness, opiates are frequently associated with adverse side effects, such as sedation, dizziness, nausea, 
vomiting, and constipation, coupled with high rates of addiction and dependence (79). Memantine is an NMDA glutamate receptor agonist that has been implicated in the development of neuropathic pain, including the development of PLP (80). Compared with a placebo, memantine reduced acute and subacute PLP after traumatic amputation in a randomized, double-blind, controlled trial and several case studies $(81,82)$. This medication, however, has not been shown to effectively treat chronic PLP $(83,84)$.

Therapeutic efforts to target the DRG have shown promise in temporarily eliminating PLP by reducing hyperexcitability of neurons, thereby prohibiting pain signals from firing $(85,86)$. Injection of lidocaine, a sodium channel blocker, into the DRG transiently relieved PLP and PLS (60). When delivered continuously via an indwelling catheter, relief of PLP and PLS could be extended for the duration of the lidocaine administration, up to 12 days in the above study, demonstrating the importance of long-term repeated blocking in the PNS as a valuable clinical tool for alleviating PLP. Although these studies were small and require further investigation, they show promise in discovering therapies that can aid in PLP relief.

MT (Figure 3A) is noninvasive and perhaps one of the least expensive and most effective modalities used for the treatment of PLP. Chan and colleagues conducted the first randomized sham-controlled MT study showing that MT was effective in reducing PLP in $93 \%$ of participants (87). Additional findings showed that amputees who practiced MT reported a larger reduction in PLP than those amputees who only mentally visualized and attempted to move their absent limbs (87), and the time to pain relief was dependent upon the starting pain level (88). A study on bilateral lower-limb amputees found reduced PLP in both phantom legs when participants viewed another person's limbs moving in the same way as their phantom legs (44). Such findings further support the role of visual feedback in modulating pain responses. A study by Foell and colleagues suggests that MT causes the somatosensory cortex of amputees to return to the baseline configuration existing before amputation (89). Further, MT has been shown to reduce PLP after BPA (where the limb is deafferented but intact), supporting the hypothesis that both the PNS and CNS interact to facilitate the reduction of PLP (31). Thus, MT may aid in the reestablishment of somatosensory cortex organization that existed before the amputation (or disconnection, in the case of BPA) (31). More work is needed, however, to elucidate the clinical efficacy of MT and the mechanisms by which this therapy alleviates PLP and lead to an understanding of why some people do not benefit from MT.
VR (Figure 3B) holds the potential to create a more "sophisticated" immersive form of MT (90). The use of advanced technology to create virtual images of amputees' missing limb(s) has demonstrated encouraging results for alleviating PLP. One study used a VR therapy with eight participants viewing a virtual image of a limb enacting various movements and replicating the movements with their phantom limbs, which resulted in an average $38 \%$ decrease in PLP (91). Seven of eight participants saw pain reduction during the intervention, with five of eight reporting more than a $30 \%$ decrease. In an effort to utilize intrinsic brain neuroplasticity, a more recent study reported pain relief in upperlimb amputees participating in biweekly augmented reality and VR (92). These results indicate that VR therapy should be further examined and compared with traditional MT.

\section{Conclusion}

Although PLP has plagued amputees for millennia, the condition still perplexes researchers today, with no universally efficacious treatment available. Further research investigating the etiology of both PLS and PLP, especially targeting PNS roles, and developing novel treatments are absolutely necessary. Investigation of the role of vision in PLP experiences is an important avenue to follow. Vision seems to play a critical role in reducing PLP in MT and VR therapies and in prosthesis usage that lacks somatosensory input. To date, there have been no studies conducted on visually impaired amputees to determine the presence or lack of PLP. In conjunction with vision, the other component that seems to be necessary in the most effective treatments is muscle activation of the residual limb. Activating the remaining muscles to complete natural movements may assist in diminishing cortical reorganization and/or connecting vision to proprioceptive sensations of movement. The efficacies of therapies that target both vision and muscle activity seem to underscore the general characterization of PLP as a complex neuropathic syndrome with PNS and CNS components.

\section{Acknowledgments}

This work was supported by start-up funds from the University of Tennessee Health Science Center to JWT.

Address correspondence to: Jack W. Tsao, Department of Neurology, University of Tennessee Health Science Center, 855 Monroe Avenue, Suite 415, Memphis, Tennessee 38163, USA. Phone: 901.448.7674; Email: jtsao@uthsc.edu.
1. Weinstein SM. Phantom limb pain and related disorders. Neurol Clin. 1998;16(4):919-936.

2. Carlen PL, Wall PD, Nadvorna H, Steinbach T. Phantom limbs and related phenomena in recent traumatic amputations. Neurology. 1978;28(3):211-217.

3. Giummarra MJ, Gibson SJ, Georgiou-Karistianis $\mathrm{N}$, Bradshaw JL. Central mechanisms in phantom limb perception: the past, present and future. Brain Res Rev. 2007;54(1):219-232.

4. Finger S, Hustwit MP. Five early accounts of phantom limb in context: Paré, Descartes, Lemos, Bell, and Mitchell. Neurosurgery. 2003;52(3):675-686; discussion 685.

5. Nathanson M. Phantom limbs as reported by S.
Weir Mitchell. Neurology. 1988;38(3):504-505.

6. Ewalt JR, Randall GC, Morris H. The phantom limb. Psychosom Med. 1947;9(2):118-123.

7. Woodhouse A. Phantom limb sensation. Clin Exp Pharmacol Physiol. 2005;32(1-2):132-134.

8. Veterans Health Initiative. Traumatic Amputation And Prosthetics: Independent Study Course. Washington, DC, USA: Department of Veterans Affairs; 2002. https://permanent.access. gpo.gov/lps77365/traumatic_amputation.pdf. Accessed March 27, 2018.

9. Ziegler-Graham K, MacKenzie EJ, Ephraim PL, Travison TG, Brookmeyer R. Estimating the prevalence of limb loss in the United States: 2005 to 2050. Arch Phys Med Rehabil. 2008;89(3):422-429.
10. Perry BN, Moran CW, Armiger RS, Pasquina PF, Vandersea JW, Tsao JW. Initial clinical evaluation of the modular prosthetic limb. Front Neurol. 2018;9:153.

11. Wartan SW, Hamann W, Wedley JR, McColl I. Phantom pain and sensation among British veteran amputees. Br J Anaesth. 1997;78(6):652-659.

12. Sherman RA, Sherman CJ, Parker L. Chronic phantom and stump pain among American veterans: results of a survey. Pain. 1984;18(1):83-95.

13. Li Z, et al. Spinal versus brain microglial and macrophage activation traits determine the differential neuroinflammatory responses and analgesic effect of minocycline in chronic neuropathic pain. Brain Behav Immun. 2016;58:107-117. 
14. Lee PM, So Y, Park JM, Park CM, Kim HK, Kim $\mathrm{JH}$. Spinal cauda equina stimulation for alternative location of spinal cord stimulation in intractable phantom limb pain syndrome: a case report. Korean J Pain. 2016;29(2):123-128.

15. Flor H, Nikolajsen L, Staehelin Jensen T. Phantom limb pain: a case of maladaptive CNS plasticity? Nat Rev Neurosci. 2006;7(11):873-881.

16. Flor $\mathrm{H}$, et al. Phantom-limb pain as a perceptual correlate of cortical reorganization following arm amputation. Nature. 1995;375(6531):482-484.

17. Livingston KE. The phantom limb syndrome. A discussion of the role of major peripheral nerve neuromas. J Neurosurg. 1945;2(3):251-255.

18. Jensen TS, Krebs B, Nielsen J, Rasmussen P. Immediate and long-term phantom limb pain in amputees: incidence, clinical characteristics and relationship to pre-amputation limb pain. Pain. 1985;21(3):267-278.

19. Flor H. Phantom-limb pain: characteristics, causes, and treatment. Lancet Neurol. 2002;1(3):182-189.

20. Melzack R. Pain and the neuromatrix in the brain. J Dent Educ. 2001;65(12):1378-1382.

21. Bekrater-Bodmann R, et al. Post-amputation pain is associated with the recall of an impaired body representation in dreams-results from a nation-wide survey on limb amputees. PLoS One. 2015;10(3):e0119552.

22. Botvinick M, Cohen J. Rubber hands 'feel' touch that eyes see. Nature. 1998;391(6669):756.

23. Ehrsson HH. The experimental induction of out-of-body experiences. Science. 2007;317(5841):1048.

24. McCabe CS, Haigh RC, Halligan PW, Blake DR. Simulating sensory-motor incongruence in healthy volunteers: implications for a cortical model of pain. Rheumatology (Oxford). 2005;44(4):509-516.

25. Sapunar D, Kostic S, Banozic A, Puljak L. Dorsal root ganglion - a potential new therapeutic target for neuropathic pain. J Pain Res. 2012;5:31-38.

26. Wall JT, Xu J, Wang X. Human brain plasticity: an emerging view of the multiple substrates and mechanisms that cause cortical changes and related sensory dysfunctions after injuries of sensory inputs from the body. Brain Res Brain Res Rev. 2002;39(2-3):181-215.

27. Merzenich MM, Kaas JH, Wall J, Nelson RJ, Sur $\mathrm{M}$, Felleman D. Topographic reorganization of somatosensory cortical areas $3 \mathrm{~b}$ and $1 \mathrm{in}$ adult monkeys following restricted deafferentation. Neuroscience. 1983;8(1):33-55.

28. Borsook D, et al. Acute plasticity in the human somatosensory cortex following amputation. Neuroreport. 1998;9(6):1013-1017.

29. Birbaumer N, et al. Effects of regional anesthesia on phantom limb pain are mirrored in change in cortical reorganization. J Neurosci. 1997;17(14):5503-5508.

30. Jensen TS, Nikolajsen L. Phantom pain and other phenomena after amputation. In: Wall PD, Melzack RA, eds. Textbook of Pain. 4th ed. Edinburgh, United Kingdom: Churchill Livingstone; 1999:799-814.

31. Tsao JW, Finn SB, Miller ME. Reversal of phantom pain and hand-to-face remapping after brachial plexus avulsion. Ann Clin Transl Neurol. 2016;3(6):463-464.

32. Ramachandran VS, Hirstein W. The perception of phantom limbs. The D. O. Hebb lecture. Brain. 1998;121(pt 9):1603-1630.

33. Chen R, Cohen LG, Hallett M. Nervous system reorganization following injury. Neuroscience. 2002;111(4):761-773.

34. Makin TR, Scholz J, Henderson Slater D, Johansen-Berg H, Tracey I. Reassessing cortical reorganization in the primary sensorimotor cortex following arm amputation. Brain. 2015;138(Pt 8):2140-2146.

35. Penfield W, Faulk M. The insula, further observation on its function. Brain. 1955;78(4):445-470.

36. Grammer GG, Williams-Joseph S, Cesar A, Adkinson DK, Spevak C. Significant reduction in phantom limb pain after low-frequency repetitive transcranial magnetic stimulation to the primary sensory cortex. Mil Med. 2015;180(1):e126-e128.

37. Ergenzinger ER, Glasier MM, Hahm JO, Pons TP. Cortically induced thalamic plasticity in the primate somatosensory system. Nat Neurosci. 1998;1(3):226-229.

38. Davis KD, Kiss ZH, Luo L, Tasker RR, Lozano AM, Dostrovsky JO. Phantom sensations generated by thalamic microstimulation. Nature. 1998;391(6665):385-387.

39. Waxman SG, Hains BC. Fire and phantoms after spinal cord injury: $\mathrm{Na}+$ channels and central pain. Trends Neurosci. 2006;29(4):207-215.

40. Li CX, Chappell TD, Ramshur JT, Waters RS. Forelimb amputation-induced reorganization in the ventral posterior lateral nucleus (VPL) provides a substrate for large-scale cortical reorganization in rat forepaw barrel subfield (FBS). Brain Res. 2014;1583:89-108.

41. Anderson-Barnes VC, McAuliffe C, Swanberg KM, Tsao JW. Phantom limb pain - a phenomenon of proprioceptive memory? Med Hypotheses. 2009;73(4):555-558.

42. Gentili ME, Verton C, Kinirons B, Bonnet F. Clinical perception of phantom limb sensation in patients with brachial plexus block. Eur J Anaesthesiol. 2002;19(2):105-108.

43. Katz J, Melzack R. Pain 'memories' in phantom limbs: review and clinical observations. Pain 1990;43(3):319-336.

44. Tung ML, et al. Observation of limb movements reduces phantom limb pain in bilateral amputees. Ann Clin Transl Neurol. 2014;1(9):633-638.

45. Ossipov MH, Dussor GO, Porreca F. Central modulation of pain. JClin Invest. 2010;120(11):3779-3877.

46. Purves D, et al. Neuroscience. 2nd ed. Sunderland, Massachusetts, USA: Sinauer Associates; 2001

47. Borghi B, et al. The use of prolonged peripheral neural blockade after lower extremity amputation: the effect on symptoms associated with phantom limb syndrome. Anesth Analg. 2010;111(5):1308-1315.

48. Halbert J, Crotty M, Cameron ID. Evidence for the optimal management of acute and chronic phantom pain: a systematic review. Clin J Pain. 2002;18(2):84-92.

49. Nyström B, Hagbarth KE. Microelectrode recordings from transected nerves in amputees with phantom limb pain. Neurosci Lett. 1981;27(2):211-216

50. Devor M. Neuropathic pain: pathophysiological response of nerves to injury. In: McMahon SB, Koltzenburg M, Tracey I, Turk DC, eds. Wall and
Melzack's Text Book of Pain. Vol 6. Philadelphia, Pennsylvania, USA: Elsevier; 2013:861-888.

51. Wu H, Sultana R, Taylor KB, Szabo A. A prospective randomized double-blinded pilot study to examine the effect of botulinum toxin type A injection versus lidocaine/depomedrol injection on residual and phantom limb pain: initial report. Clin J Pain. 2012;28(2):108-112.

52. Navarro X, Vivó M, Valero-Cabré A. Neural plasticity after peripheral nerve injury and regeneration. Prog Neurobiol. 2007;82(4):163-201.

53. Devor M. Neuropathic pain: what do we do with all these theories? Acta Anaesthesiol Scand. 2001;45(9):1121-1127.

54. Woolf CJ, Thompson SW. The induction and maintenance of central sensitization is dependent on N-methyl-D-aspartic acid receptor activation; implications for the treatment of post-injury pain hypersensitivity states. Pain . 1991;44(3):293-299.

55. Pearson JA, Richardson TL. The influence of stimulus intensity on sensitization of the flexor reflex. Exp Neurol. 1975;47(1):194-197.

56. Zhang XL, Albers KM, Gold MS. Inflammation-induced increase in nicotinic acetylcholine receptor current in cutaneous nociceptive DRG neurons from the adult rat. Neuroscience. 2015;284:483-499.

57. Pagadala P, et al. Loss of NR1 subunit of NMDARs in primary sensory neurons leads to hyperexcitability and pain hypersensitivity: involvement of $\mathrm{Ca}(2+)$-activated small conductance potassium channels. J Neurosci. 2013;33(33):13425-13430.

58. Sato K, Kiyama H, Park HT, Tohyama M. AMPA, KA and NMDA receptors are expressed in the rat DRG neurones. Neuroreport. 1993;4(11):1263-1265.

59. Du X, et al. Local GABAergic signaling within sensory ganglia controls peripheral nociceptive transmission. JClin Invest. 2017;127(5):1741-1756.

60. Vaso A, et al. Peripheral nervous system origin of phantom limb pain. Pain. 2014;155(7):1384-1391.

61. Devor M. Sodium channels and mechanisms of neuropathic pain. J Pain. 2006;7(1 suppl 1):S3-S12.

62. Waxman SG, Cummins TR, Dib-Hajj SD, Black JA. Voltage-gated sodium channels and the molecular pathogenesis of pain: a review. J Rehabil Res Dev. 2000;37(5):517-528.

63. Waxman SG, Dib-Haij S, Cummins TR, Black JA. Sodium channels and pain. Proc Natl Acad Sci US A. 1999;96(14):7635-7639.

64. Du X, Gamper N. Potassium channels in peripheral pain pathways: expression, function and therapeutic potential. Curr Neuropharmacol. 2013;11(6):621-640.

65. Tsantoulas C, et al. Sensory neuron downregulation of the Kv9.1 potassium channel subunit mediates neuropathic pain following nerve injury. J Neurosci. 2012;32(48):17502-17513.

66. Herzberg U, Eliav E, Dorsey JM, Gracely RH, Kopin IJ. NGF involvement in pain induced by chronic constriction injury of the rat sciatic nerve. Neuroreport. 1997;8(7):1613-1618.

67. McLachlan EM, Jänig W, Devor M, Michaelis M. Peripheral nerve injury triggers noradrenergic sprouting within dorsal root ganglia. Nature. 1993;363(6429):543-546.

68. Li S, et al. The transcriptional landscape of dorsal 
root ganglia after sciatic nerve transection. Sci Rep. 2015;5:16888.

69. Bach S, Noreng MF, Tjéllden NU. Phantom limb pain in amputees during the first 12 months following limb amputation, after preoperative lumbar epidural blockade. Pain. 1988;33(3):297-301.

70. Nikolajsen L, Ilkjaer S, Krøner K, Christensen JH, Jensen TS. The influence of preamputation pain on postamputation stump and phantom pain. Pain. 1997;72(3):393-405.

71. Karl A, Mühlnickel W, Kurth R, Flor H. Neuroelectric source imaging of steady-state movement-related cortical potentials in human upper extremity amputees with and without phantom limb pain. Pain. 2004;110(1-2):90-102.

72. Yanagisawa T, et al. Induced sensorimotor brain plasticity controls pain in phantom limb patients. Nat Commun. 2016;7:13209.

73. Preißler S, Dietrich C, Blume KR, Hofmann GO, Miltner WH, Weiss T. Plasticity in the visual system is associated with prosthesis use in phantom limb pain. Front Hum Neurosci. 2013;7:311.

74. Hagura N, et al. Activity in the posterior parietal cortex mediates visual dominance over kinesthesia. J Neurosci. 2007;27(26):7047-7053.

75. Levendoglu F, Ogün CO, Ozerbil O, Ogün TC, Ugurlu H. Gabapentin is a first line drug for the treatment of neuropathic pain in spinal cord injury. Spine. $2004 ; 29(7): 743-751$.

76. Wu CL, et al. Analgesic effects of intravenous lidocaine and morphine on post amputation pain. Anesthesiology. 2002;96(4):841-848.

77. Wu CL, et al. Morphine versus mexiletine for treatment of post amputation pain. Anesthesiolo- gy. 2008;109(2):289-296.

78. Huse E, Larbig W, Flor H, Birbaumer N. The effect of opioids on phantom limb pain and cortical reorganization. Pain. 2001;90(1-2):47-55.

79. Benyamin R, et al. Opioid complications and side effects. Pain Physician. 2008;11(2 suppl):S105-S120.

80. Schley M, et al. Continuous brachial plexus blockade in combination with the NMDA receptor antagonist memantine prevents phantom pain in acute traumatic upper limb amputees. Eur J Pain. 2007;11(3):299-308.

81. Hackworth RJ, Tokarz KA, Fowler IM, Wallace SC, Stedje-Larsen ET. Profound pain reduction after induction of memantine treatment in two patients with severe phantom limb pain. Anesth Analg. 2008;107(4):1377-1379.

82. Neil M, Dale M, Gillespie G. Successful use of Memantine in the treatment of severe phantom limb pain: case report and literature review. Scott Med J. 2010;55(2):58.

83. Wiech $\mathrm{K}$, et al. A placebo-controlled randomized crossover trial of the N-methyl-D-aspartic acid receptor antagonist, memantine, in patients with chronic phantom limb pain. Anesth Analg. 2004;98(2):408-413, table of contents.

84. Maier C, et al. Efficacy of the NMDA-receptor antagonist memantine in patients with chronic phantom limb pain--results of a randomized double-blinded, placebo-controlled trial. Pain. 2003;103(3):277-283.

85. Amir R, Michaelis M, Devor M. Burst discharge in primary sensory neurons: triggered by subthreshold oscillations, maintained by depolarizing after potentials. JNeurosci. 2002;22(3):1187-1198.
86. Eldabe S, et al. Dorsal root ganglion (DRG) stimulation in the treatment of phantom limb pain (PLP). Neuromodulation. 2015;18(7):610-616; discussion 616.

87. Chan BL, et al. Mirror therapy for phantom limb pain. NEngl JMed. 2007;357(21):2206-2207.

88. Griffin SC, et al. Trajectory of phantom limb pain relief using mirror therapy: retrospective analysis of two studies. Scand J Pain. 2017;15:98-103.

89. Foell J, Bekrater-Bodmann R, Diers M, Flor H. Mirror therapy for phantom limb pain: brain changes and the role of body representation. Eur J Pain. 2013;18(5):729-739.

90. Ortiz-Catalan M, Sander N, Kristoffersen MB, Håkansson B, Brånemark R. Treatment of phantom limb pain (PLP) based on augmented reality and gaming controlled by myoelectric pattern recognition: a case study of a chronic PLP patient. Front Neurosci. 2014;8:24.

91. Mercier C, Sirigu A. Training with virtual visual feedback to alleviate phantom limb pain. Neurorehabil Neural Repair. 2009;23(6):587-594.

92. Ortiz-Catalan M, et al. Phantom motor execution facilitated by machine learning and augmented reality as treatment for phantom limb pain: a single group, clinical trial in patients with chronic intractable phantom limb pain. Lancet. 2016;388(10062):2885-2894.

93. Giraux P, Sirigu A, Schneider F, Dubernard JM. Cortical reorganization in motor cortex after graft of both hands. Nat Neurosci. 2001; 4(7): 691-692.

94. Ramachandran VS, Rogers-Ramachandran D. Synaesthesia in phantom limbs induced with mirrors. Proc Biol Sci. 1996;263(1369):377-386. 